\title{
Canadian Urological Association clinical practice guideline on testosterone deficiency in men: Evidence-based Q\&A
}

Ethan D. Grober, $\mathrm{MD}^{1}$; Yonah Krakowsky, $\mathrm{MD}^{2}$; Mohit Khera, MD 3 ; Daniel T. Holmes, MD; Jay C. Lee, MD ${ }^{5}$; John E. Grantmyre, $\mathrm{MD}^{6}$; Premal Patel, $\mathrm{MD}^{7}$; Richard A. Bebb, MD ${ }^{8}$; Ryan Fitzpatrick, $\mathrm{MD}^{9}$; Jeffrey D. Campbell, $\mathrm{MD}^{10}$; Serge Carrier, $\mathrm{MD}^{11}$; Abraham Morgentaler, $\mathrm{MD}^{12}$

${ }^{1}$ Division of Urology, Women's College Hospital \& Sinai Health System, Department of Surgery, University of Toronto, Toronto, ON, Canada; ${ }^{2}$ Division of Urology, Women's College Hospital \& Sinai Health System, Department of Surgery, University of Toronto, Toronto, ON, Canada; ${ }^{3}$ Scott Department of Urology, Baylor College of Medicine, Houston, TX, United States; ${ }^{4}$ Department of Pathology \& Laboratory Medicine, University of British Columbia, Vancouver, BC, Canada; ${ }^{5}$ Division of Urology, Department of Surgery, University of Calgary, Calgary, AB, Canada; ${ }^{6}$ Department of Urology, Dalhousie University, Halifax, NS, Canada; ${ }^{7}$ Division of Urology, Department of Surgery, University of Manitoba, Winnipeg, MB, Canada; ${ }^{8}$ Division of Endocrinology \& Department of Urological Sciences, University of British Columbia, Vancouver, BC, Canada; ${ }^{9}$ Division of Urology, Women's College Hospital \& Sinai Health System, Department of Surgery, University of Toronto, Toronto, ON, Canada; ${ }^{10}$ Division of Urology, Department of Surgery, University of Western Ontario, London, ON, Canada

${ }^{11}$ Division of Urology, Department of Surgery, McGill University, Montreal, QC, Canada; ${ }^{12}$ Department of Urology, Harvard Medical School, Boston, MA, United States

Cite as: Grober ED, Krakowsky Y, Khera M, et al. Canadian Urological Association clinical practice guideline: Testosterone deficiency in men - evidence-based Q\&A. Can Urol Assoc J 2021 February 23; Epub ahead of print. http://dx.doi.org/10.5489/cuaj.7252

Published online February 23, 2021

$* * *$

\section{Introduction}

As part of their ongoing commitment to education and best practice standards, the Canadian Urological Association (CUA) solicited the creation of a clinical practice guideline dedicated to testosterone deficiency (TD) in men.

Modern androgen therapy essentially began when testosterone was chemically synthesized in 1935. ${ }^{1}$ This scientific discovery (along with the discovery of other sex hormones - estrogen and progesterone) was awarded the Nobel Prize in Chemistry in 1939. ${ }^{2}$ Throughout most of its history, the concept of TD and replacement has been associated with confusion and controversy. Concerns relating to prostate and cardiovascular health, performance enhancement via steroid abuse and the perception that TD is simply part of the "normal" process of aging has resulted in reluctance of many healthcare providers to diagnose and treat the condition. ${ }^{3}$ 
In 2013, a comprehensive Canada-wide needs assessment revealed that over 25\% of Canadian physicians were uncomfortable with the diagnosis and treatment of TD and identified significant knowledge gaps related to patient management. ${ }^{4}$ To address the knowledge gaps, the Canadian Clinical Practice Guideline on the Diagnosis and Management of Testosterone Deficiency Syndrome in Adult Males was published in the CMAJ. ${ }^{5}$ As part of its mandate, the guideline was offered as a "living document," to be updated periodically in a perpetual effort to incorporate advances in scientific and clinical discovery. The current CUA guideline was approached in this spirit and serves as an educational tool that builds on existing knowledge and offers a contemporary update to the literature.

\section{Methods}

The current guideline is structured as a series of Questions \& Answers (Q\&A) relating to the diagnosis and management of TD. By way of this structure, the guideline offers a practical, evidence-based overview and recommendations related to the diagnosis, treatment, monitoring, risks and benefits of TD and testosterone therapy. It was designed to be a straightforward and user-friendly guide for specialists (urologists, endocrinologists, psychiatrists, geriatricians), general practitioners and the patients they treat. The guideline offers Canadian-specific content with respect to knowledge and attitudes related to TD, laboratory diagnostic testing, Health Canada approved treatment formulations and funding considerations.

The authorship group represents a panel of Canadian and international experts in the fields of men's health and TD. Representation is inter-provincial and multi-disciplinary covering the fields of urology, internal medicine/endocrinology and clinical biochemistry.

Relevant publications on TD and testosterone therapy were sought using a combination of Medline, EMBASE, the Cochrane Central Register of Controlled Trials (CENTRAL) and PubMed searches. The search strategy involved a series of key words including: testosterone deficiency, hypogonadism, low testosterone, androgen deficiency, andropause and testosterone replacement therapy. Filters included English language, human studies and an index date up to December 1, 2020. Recently published guidelines from the Canadian Men's Health Foundation, American Urological Association, The Endocrine Society and the European Association of Urology were included as supportive, summative content. ${ }^{5-9}$

Whenever possible, guideline statements and recommendations have been assigned levels of evidence (LE) based on the GRADE Working Group framework (LE: high, moderate, low, very low quality of evidence). ${ }^{10}$ In brief, randomized controlled trials were initially rated as highquality and observational studies as low-quality evidence. Additionally, the strength or importance of the recommendations was also graded as either "strong" or "weak" based on the quantity, quality, and consistency of the evidence available.

Following a systematic search, panel members were asked to review the identified literature and existing guidelines in an effort to highlight areas appropriate for an evidence-based review 
and update. ${ }^{11}$ Additionally, each member was surveyed and independently tasked to identify a series of fundamental questions relating to TD that should be addressed or updated as part of the guideline (i.e. common questions that colleagues ask of each other and patients ask of their physicians). Submissions were collated and common themes were identified. The proposed list of questions was collectively reviewed by panel members and finalized through consensus. Questions were assigned to each author based on clinical expertise for an evidence-based Answer. Responses were collectively reviewed by all panel members for feedback, clarification and consensus.

\section{Evidenced-based Q\&A}

\section{Practical overview}

1. What is the definition of testosterone deficiency?

Testosterone deficiency is a clinical and biochemical syndrome that may occur in men in association with advancing age, however younger men with certain conditions may also be affected. The condition is characterized by deficient testicular production of testosterone, with or without changes in receptor sensitivity to androgens. It may affect multiple organ systems and can result in significant health consequences and a negative impact on quality of life. ${ }^{5,12}$

\section{What is the prevalence of testosterone deficiency among Canadian men?}

The prevalence of TD varies between populations and is difficult to determine in the absence of wide-spread, routine testing which is currently not the standard of care. Current studies evaluating the true prevalence of TD are conflicting due to variable reporting measures, testosterone level cut-offs and testing protocols. The crude Canadian prevalence of biochemical TD is estimated to be approximately $25 \%$ among men aged $40-62$ years. ${ }^{13}$ The rate of TD increases with age. Appreciating that prevalence rates will vary based on case definitions and biochemical testosterone thresholds, the prevalence of TD is estimated to be $4-12 \%$ in men between age $50-59$ years, $9-23 \%$ in men aged $60-69$ years, and $28-49 \%$ in men over 70 years old. ${ }^{6,14,15}$

\section{What are the common signs and symptoms of testosterone deficiency?}

Testosterone deficiency may present with a variety of clinical manifestations including sexual, cognitive and physical/structural signs and symptoms (Table 1). Common signs of TD include decreased libido and vitality, sexual dysfunction, fatigue and/or mood changes. ${ }^{5,16}$ The signs and symptoms of TD are not exclusive to this syndrome and are commonly associated with other conditions related to male aging. As such, the clinical presentation should be correlated with biochemical confirmation of low/equivocal serum testosterone levels. ${ }^{6}$ 


\section{Why does testosterone deficiency occur?}

Testosterone production primarily occurs in the Leydig cells of the testis and is regulated by the hypothalamic-pituitary-gonadal-axis (HPG, Figure 1). ${ }^{17}$ For normal testosterone production, gonadotropin releasing hormone $(\mathrm{GnRH})$ is released in a pulsatile manner from the hypothalamus. GnRH stimulates the production and release of luteinizing hormone (LH) and follicle stimulating hormone (FSH) from the anterior pituitary. LH stimulates testosterone production by the Leydig cells in the testis. The HPG axis is regulated by a negative feedback loop whereby high levels of testosterone send a feedback signal (via estradiol and testosterone) to both the pituitary and the hypothalamus to inhibit ongoing testosterone production. ${ }^{17}$ This pathway is essential for testosterone production and any disruptions can cause downstream effects and inhibit the production of testosterone.

Clinical TD results when the body fails to produce adequate levels of testosterone. If there is an intrinsic failure at the level of the testis to produce testosterone, it is termed primary hypogonadism. By contrast, if there is a hormonal/gonadotropin signaling a problem within the brain (hypothalamus or pituitary), it is considered secondary hypogonadism. Primary hypogonadism results in low levels of testosterone but high levels of gonadotropins (LH, FSH). The brain is trying to amplify its signal to the testis to produce testosterone. Secondary hypogonadism is a result of a neural signaling deficiency and clinically manifests with low levels of testosterone and low or normal levels of gonadotropins. A combined pattern of primary and secondary hypogonadism is common. TD or hypogonadism occurs as men age due to several factors including a decrease in Leydig cell size, reduced Leydig cell sensitivity to LH, increases in systemic estrogen levels and a diminution of GnRH pulsatility. ${ }^{18,19}$ Multiple medications and medical conditions are also responsible for TD (Table 2).

\section{Diagnosis}

5.How best to establish the diagnosis of testosterone deficiency with history \& physical examination?

The diagnosis of TD is established through the collective findings from history, physical examination and serum testosterone measurements (Level of Evidence (LE)-moderate, strong recommendation). The clinical manifestations of TD are non-specific with recognized variability from patient to patient. Table 1 lists clinical signs and/or symptoms commonly associated with testosterone deficiency and the medical history should be focused towards identifying the cognitive, sexual and physical/structural manifestations of TD. ${ }^{5-7,9}$

Physical examination should be undertaken with particular attention to the following testosterone dependent tissues and organs.

- Testicles (size, firmness, location) 
- Penile development (length, location of urethral opening)

- Prostate size and consistency by performing a digital rectal examination (DRE)

- Secondary sex characteristics: facial and body hair, changes with puberty's[épi

- Breast tissue development (gynecomastia) s[epe

- Weight and fat distribution

- Muscle mass and bone structure/function

6.Are there valid screening questionnaires for testosterone deficiency? Are they helpful in establishing the diagnosis?

Several validated screening questionnaires for TD exist including the Androgen Deficiency in the Aging Male (ADAM), Quantitative ADAM, Aging Males' Symptoms Scale, Massachusetts Male Aging Study Questionnaire as well as questionnaires that focus on a specific common element of testosterone deficiency (i.e. erectile dysfunction): International Index of Erectile Function, Sexual Health Inventory for Men and Erection Hardness Score. ${ }^{13,20-24}$

Due to their lack of specificity (approximately 20\%), screening questionnaires alone should not be substituted for a detailed history, physical examination and biochemical testing when establishing the diagnosis of TD. ${ }^{6,9,25}$ Standardized questionnaires are often helpful for academic/research purposes and can act as an initial screen to facilitate discussion and highlight areas of focus for further evaluation (LE-low, weak recommendation).

7.What is the recommended laboratory test to diagnose testosterone deficiency? Challenges have always existed in the quality of commercial immunoassays to accurately measure testosterone relative to validated reference methodologies - the gold standards being equilibrium dialysis and mass spectrometry. ${ }^{26}$ The development of the Centre for Disease Controls Hormone Standardization (HoSt) program (https://www.cdc.gov/labstandards/hs_standardization.html) has forced many commercial immunoassay vendors to improve their calibration and accuracy in the measurement of testosterone. $^{27}$ Total testosterone (morning draw: $7 \mathrm{am}-11 \mathrm{am}$ ) remains the best initial screening test to diagnose TD. ${ }^{5,6}$ Calculated free or bioavailable testosterone based on age-dependant standards of total testosterone, sex hormone binding globulin (SHBG) and albumin concentrations can be determined to resolve equivocal total testosterone measures in symptomatic men. ${ }^{5}$ (LE-moderate, strong recommendation)

\section{What is the biochemical level or cut off to diagnose testosterone deficiency?}

The clinical diagnosis of TD relies on the correlation of recognized signs and symptoms with biochemical confirmation of deficient serum testosterone concentrations. Establishing an absolute biochemical cut-off diagnostic for TD is challenging due to several factors including: limitations in measurement accuracy; laboratory and immunoassay variability; lack of agerelated reference standards; unknown baseline testosterone levels in individual patients; and 
variable target organ receptor sensitivity to circulating testosterone concentrations. Most clinician experts and previously published guidelines would suggest that a total testosterone $<10$ $\mathrm{nmol} / \mathrm{L}$ represents a reasonable diagnostic threshold consistent for TD, while appreciating that some patients may manifest symptoms of TD at higher levels. ${ }^{5,6,12}$ Testosterone measures can be used as a complimentary tool (as opposed to an absolute or diagnostic one), to support the diagnosis of TD in the context of characteristic signs and symptoms identified by way of a detailed clinical evaluation (LE-low, weak recommendation).

9.Besides measuring testosterone, what adjunctive laboratory testing is indicated? In patients with established TD, clinicians should measure serum luteinizing hormone levels (LH) to help classify the testosterone deficiency as primary (high LH) or secondary (low/normal LH). ${ }^{5,6,12}$

Serum prolactin levels should be measured in patients suspected of having secondary testosterone deficiency (low testosterone levels combined with low or low/normal LH levels). Patients with persistently high prolactin levels of unknown etiology should undergo evaluation for endocrine disorders and to rule out a prolactinoma. ${ }^{6}$

Serum estradiol should be measured in testosterone deficient patients who present with breast symptoms or gynecomastia prior to the commencement of testosterone therapy. ${ }^{6}$

(LE-moderate, strong recommendation)

10. What are potentially reversible causes of testosterone deficiency that physicians should consider?

Prior to initiating testosterone therapy, physicians should consider and manage any potentially reversible causes of $\mathrm{TD}^{5}$ (LE-moderate, strong recommendation). These may include:

- Acute illness

- Obesity

- Medication use (i.e. opioids, glucocorticoids)

- Extreme nutritional deficiencies and anorexia

- Extreme exercise

- Sleep Apnea

- Hyperprolactinemia

- Hemochromatosis

- Hypothalamic and pituitary disorders (surgery, trauma, radiation, infiltrative disorders)

Following treatment or stabilization of these conditions, reassessment of serum testosterone levels and the signs and symptoms of TD is recommended. 
11. What are the common co-morbid conditions associated with testosterone deficiency? Testosterone deficiency is associated with several specific health conditions that may prompt investigation and management of serum testosterone levels to validate such associations and compliment the management of such conditions. ${ }^{5,7}$

- Type 2 diabetes \& insulin resistance

- Metabolic syndrome

- Obesity

- Osteopenia/osteoporosis

- COPD

- Sleep Apnea

- HIV/AIDS

\section{Treatment}

12.What are the goals and benefits of testosterone therapy?

The primary goals of testosterone therapy are to mitigate the negative signs and symptoms of testosterone deficiency and improve quality of life while achieving eugonadal (normal) levels of testosterone. ${ }^{5}$

The benefits of treatment will vary according to the symptom complex of each patient. The Testosterone Trials - the largest placebo-controlled, double-blinded, multicenter, randomized controlled trial of testosterone therapy in symptomatic hypogonadal older men - demonstrated significant improvements in sexual activity, sexual desire, erectile function, mood and depressive symptoms, anemia and bone mineral density among men receiving testosterone therapy. ${ }^{28,29}$

13. What are the current treatment options for testosterone deficiency in Canada?

Table 3 summarizes the testosterone treatment formulations currently approved by Health Canada.

Compounded testosterone products are available at many compounding and online pharmacies within Canada, however published data has demonstrated significant variability of testosterone concentrations within such products leading to concerns regarding efficacy and safety. ${ }^{30}$

The choice testosterone therapy and route of administration should be a topic of discussion between the physician and the patient using a shared decision-making approach. Factors influencing this choice include safety, efficacy, tolerability, availability, preference and cost/insurance coverage. 
14. What is the recommended approach to treating a patient with characteristic symptoms of testosterone deficiency with a "normal" testosterone level?

Patients may present with a clinical picture consistent with TD, yet serum testosterone concentrations within the "normal" reference range. Efforts should be made to rule out conditions with an overlapping symptom complex (i.e. depression, hypothyroidism, sleep disorders).

Challenges in the measurement of serum testosterone, inconsistent normal reference ranges between laboratories, the lack of a "baseline" reference level of testosterone earlier in a man's life, variable androgen receptor sensitivity to a given concentration of testosterone and the ability of elevated sex-hormone binding globulin (SHBG) levels to effectively neutralize circulating testosterone may account for a symptomatic presentation with normal total serum testosterone levels. In equivocal cases, measurement of SHBG and calculation of free and/or bioavailable testosterone may provide additional insight upon which to better interpret testosterone concentrations and rationalize treatment. ${ }^{7,27,31}$

Recognizing such limitations, a supervised trial of testosterone therapy (3-months) with close monitoring of both the symptomatic and biochemical response to treatment is recommended. ${ }^{5}$ (LE-low, weak recommendation)

15. What is the recommended approach to treating a patient with NO symptoms of testosterone deficiency but a "low" testosterone level?

In general, hypogonadal men who are asymptomatic with no impact on quality of life should not be offered testosterone therapy. Investigation and consideration of treatment should be initiated in men with unexplained anemia or sarcopenia, chronic use of glucocorticoid or opioid therapy, as well as patients with human immunodeficiency virus (HIV) experiencing weight loss, irrespective of the presence of other symptoms of TD. ${ }^{5,7}$ (LE-moderate, weak recommendation)

16. What is the likelihood that my patient will respond/benefit from treatment?

Clinical response rates may vary according to several factors including baseline level of testosterone, the choice of testosterone therapy, dosage and delivery method as well as the nature and severity of each individual patient's symptoms. Overall, testosterone therapy has been shown to effectively normalize serum testosterone concentrations in the majority $(>90 \%)$ of treated men. ${ }^{29,32}$ Due to the varied clinical spectrum associated with TD and the limitations associated with objective measurement of symptoms, at the present time there is insufficient data to specifically quantify what proportion of hypogonadal men overall will respond symptomatically to treatment, and if any specific characteristics make a patient more likely to benefit. ${ }^{6}$ As such, practitioners are encouraged to concurrently monitor both patient-specific symptomatic responses from baseline as well as changes in serum testosterone levels while on treatment. Patients with a favorable biochemical and symptomatic response to treatment should continue with therapy. An alternative diagnosis should be considered in patients who fail to 
respond symptomatically despite normalization of testosterone levels and an adequate trial of therapy - approximately 3 months. (LE-low, weak recommendation)

17. What is the best treatment approach to a patient with testosterone deficiency who is interested in fertility preservation?

Many patients and clinicians are unaware that exogenous testosterone therapy can serve as a contraceptive and result in depressed sperm production leading to male infertility. ${ }^{33,34} \mathrm{~A}$ recent survey of urologists found that roughly $25 \%$ would treat infertile males with testosterone while they pursued a pregnancy. ${ }^{34}$ Exogenous testosterone administration results in negative feedback (mainly via testosterone converted to estradiol) to the pituitary leading to suppression of the primary signals for sperm (FSH) and testosterone ( $\mathrm{LH})$ production at the testicular level (Figure 1). ${ }^{17}$ Hypogonadal patients who are interested in fertility preservation should be cautioned against the isolated use of exogenous testosterone and instead pursue treatments that increase endogenous serum testosterone production (LE-high, strong recommendation). Human chorionic gonadotropin (HCG), selective estrogen receptor modulators (SERMs - clomiphene, tamoxifen) and aromatase inhibitors (AIs - anastrozole, letrozole, testolactone) are alternative treatment options (off-label) to increase endogenous serum testosterone levels without threatening fertility potential. $5,6,33,35,36$

As a unique short-acting formulation, testosterone nasal gel (Natesto) may result in less suppression of gonadotropins (LH/FSH) and semen parameters and may represent a treatment option for symptomatic hypogonadal men who desire to maintain fertility potential. ${ }^{37,38}$ Consultation with a male fertility specialist is recommended.

18. What is the suggested level of testosterone to achieve while on treatment?

Treating physicians should aim to prescribe the minimal required dosing to improve serum testosterone and hypogonadal symptoms. Recognizing the variations in normal ranges among the various laboratories throughout Canada, the current recommendations are to target correction of serum levels to the mid-normal reference range (total testosterone 14-17 nmol/L, LE-low, weak recommendation). ${ }^{5,39}$. Patients without significant symptomatic improvement and serum testosterone levels measured below the mid-normal range can be considered for dose escalation to improve clinical efficacy. Clinical experience supports raising testosterone into the upper third of the normal range for patients who do not respond symptomatically at lower levels. ${ }^{6}$ Patients with satisfactory resolution of their symptoms, but serum testosterone below the recommended target range typically do not require dose adjustment. ${ }^{28}$ Dose reductions should be prescribed in patients with serum testosterone concentrations consistently measuring above the normal range while on treatment.

19. What is the evidence for herbal or natural testosterone "boosters" in treating testosterone deficiency? 
Current evidence-based reviews of the most common ingredients in testosterone-boosters (Tboosters) on the market have found minimal to no evidence demonstrating their efficacy. ${ }^{40}$ Many of the ingredients within such compounds have never been tested for safety and/or efficacy in human trials. Among human trials, only 30\% demonstrated an improvement in testosterone levels, $2 \%$ resulted in a decrease in testosterone and $68 \%$ had either an indeterminant effect or no effect at all on testosterone levels. ${ }^{41}$ Concerningly, several case studies have demonstrated severe adverse events from T-boosters that contain banned/illicit substances including steroids. ${ }^{42,43}$ Currently, T-boosters cannot be recommended by health practitioners and patients should be cautioned regarding the unknown efficacy and safety of T-boosters along with the possibility for severe adverse events. Regulated, evidence-based treatments should be offered as an alternative in symptomatic hypogonadal men (LE-moderate, strong recommendation).

20. Aside from testosterone, are there other treatments that can be used to treat testosterone deficiency? Similar to other chronic conditions, lifestyle modification may have a role in managing TD (LElow, weak recommendation). A bidirectional relationship between obesity and testosterone levels has been demonstrated. Mechanistically, obesity lowers testosterone levels due to reductions in LH release and greater aromatization of testosterone to estradiol within adipose tissue. ${ }^{44,45}$ Weight loss, dietary restriction and bariatric surgery all have been shown to significantly increase serum testosterone levels. ${ }^{46,47}$ Exercise, including resistance training, has been shown to result in relatively modest increases in serum testosterone levels. ${ }^{48,49}$ Finally, sleep patterns have shown to influence testosterone concentrations with persistent sleep deprivation/restriction lowering testosterone levels by approximately $10-15 \%{ }^{50,51}$ A commitment to structured sleep program may negate the impact of limited sleep on testosterone concentrations. Some selective symptoms of TD can be managed with symptom specific treatments (i.e. PDE5 inhibitors for erectile dysfunction). ${ }^{52}$

\section{Treatment risks}

21.Does testosterone replacement therapy increase the risk of prostate cancer?

Despite historical teachings about the relationship between testosterone on prostate cancer biology and risk, there is now consistent evidence that testosterone therapy does not increase a man's risk for developing prostate cancer. In the largest randomized controlled trial (RCT) to date (The Testosterone Trials), involving 790 men 65 years and older treated for 12-months with either testosterone gel or placebo, despite close surveillance, only one man developed prostate cancer during the course of the study. ${ }^{29}$ Supporting such observations, a meta-analysis of 22 RCTs involving 2351 men revealed no greater risk of developing prostate cancer among those who received testosterone therapy compared with those who received placebo. ${ }^{53}$ There is no 
association between the risk prostate cancer and serum testosterone concentrations. Men with the highest naturally occurring (endogenous) testosterone levels, do not exhibit significantly higher prostate specific antigen (PSA) levels or a greater subsequent risk developing prostate cancer than men with the lowest levels of endogenous testosterone. ${ }^{54,55}$ Finally, the evolving literature suggests among appropriately-selected hypogonadal men with prostate cancer managed with active surveillance, radical prostatectomy or radiation therapy, testosterone therapy does not appear to significantly increase the risk of biochemical (PSA) progression, local or metastatic progression, overall mortality and cancer-specific mortality. ${ }^{56-58}$

Symptomatic men with TD who have been diagnosed with localized prostate cancer and treated (surgery, radiation) or followed with active surveillance without evidence of active disease can considered for a medically supervised trial of testosterone therapy (LE-low, weak recommendation). Consultation with a urologic specialist is recommended. Patients with metastatic or high-risk prostate cancer who are likely to require androgen deprivation therapy should not be offered testosterone therapy (LE-moderate, strong recommendation). ${ }^{5}$

22.Does testosterone replacement therapy increase the risk of benign prostatic hyperplasia $(\mathrm{BPH})$ progression and lower urinary tract symptom (LUTS)?

A collective review of the contemporary evidence to date suggests that testosterone therapy does not increase the risk of urinary symptoms due to BPH. ${ }^{59-61}$ Consistent with previous metaanalyses, in the Testosterone Trials, the largest RCT to date, involving 790 men aged 65 years and older, a similar rate of worsening urinary symptoms, suggestive of $\mathrm{BPH}$, were noted over the 1-year study period for those who received testosterone therapy compared with those who received placebo. ${ }^{29}$ Moreover, in a review of 16 RTCs involving 1030 men, testosterone therapy had no significant impact on prostate volume. ${ }^{61}$ Several studies have demonstrated improvement in urinary symptoms, peak urinary flow rates and voided volumes with the initiation of testosterone treatment in hypogonadal men which is theoretically related to its impact on muscular (bladder detrusor) function. ${ }^{59,60,62,63}$

While individual responses to any treatment, including testosterone vary, evidence suggest that testosterone therapy does not generally increase the risk of worsening voiding symptoms. As such, testosterone therapy can safely be administered to men with BPH and lower urinary tract symptoms (LE-moderate, weak recommendation).

23.Does testosterone replacement therapy increase the risk of cardiovascular disease? Evidence suggests that low endogenous (natural) levels of testosterone are associated with increased cardiovascular risk including higher rates of obesity, diabetes, dyslipidemia, metabolic syndrome and demonstrates an increased risk of cardiovascular death. ${ }^{64-66}$

The published literature on the impact of testosterone therapy on cardiovascular risk is conflicting and continues to evolve. Prior to 2010, the majority of studies and large metaanalyses suggests cardiovascular benefits among hypogonadal men initiated on testosterone 
therapy including: improved lean muscle mass, deceased total body fat and waist circumference, improved glycemic control (hemoglobin A1C levels), decreased cardiovascular events and decreased mortality. ${ }^{67-69}$

Following 2010, a series of high-impact publications raised concerns regarding the use of testosterone therapy and an increased risk cardiovascular events including myocardial infarction, stroke and death. ${ }^{70,71}$ Despite significant methodological concerns with study design and reporting, in 2014 the Food and Drug Administration (FDA) and Health Canada released warnings regarding the use of testosterone therapy and the possible risk of cardiovascular events. ${ }^{72,73}$ These warning garnered significant media and medical attention.

To date, there are no large, long-term placebo-controlled trials to help make definitive statements on testosterone therapy and cardiovascular risk. The literature available suggests that untreated testosterone deficient men are at increased risk of heart disease, cardiovascular events and death. ${ }^{64-66}$ Based on the best available evidence, symptomatic hypogonadal men with stable cardiovascular disease remain candidates for a medically supervised trial of testosterone therapy. ${ }^{5}$ An individualized risk-benefit assessment is prudent (LE-low, weak recommendation).

24. What are the contraindications to testosterone therapy?

Contraindications to testosterone therapy include: ${ }^{5-8,11}$

- Allergy or hypersensitivity to prescribed treatment

- Known or suspected male breast cancer

- Patients who desire fertility preservation (refer to Question and Answer \#17)

- Patients with metastatic or high-risk prostate cancer who are likely to require androgen deprivation therapy

- Patients with unstable cardiovascular disease

\section{Monitoring}

25. What monitoring is required for a patient receiving testosterone therapy? After initiating testosterone therapy, patients should have regular monitoring for response to treatment and adverse events. Table 4 summarizes an evidence-based monitoring schedule for patients on testosterone therapy. ${ }^{5-7,9}$

Adjustments to treatment dose and delivery can be made based on patient response, adverse events, and bloodwork results. Testosterone levels (+/- gonadotropins) should be measured to determine adherence and absorption of treatment (LE-moderate, strong recommendation). ${ }^{5,6}$ The recommended timing of testosterone measurement relative to the last treatment dose will vary depending on the testosterone formulation (i.e. mid-cycle for injectables) and patients should be counselled appropriately to ensure accurate interpretation of results and response to treatment.

A patient's hematocrit should be followed to ensure they are not developing polycythemia. Polycythemia is a common side effect of testosterone therapy and may be associated with 
thromboembolic events (LE-moderate, strong recommendation). ${ }^{69,74}$ Management strategies for persistent treatment induced polycythemia (hematocrit levels $55 \%$ or greater) may include a dose reduction of testosterone (concentration or application frequency), drug holiday, formulation change or blood donation/phlebotomy.

Prostate specific antigen (PSA) testing and digital rectal examination should be performed to monitor prostate health in accordance with the evidence-based guidelines for prostate cancer screening (LE-low, weak recommendation). ${ }^{75}$ The Canadian Urological Association guideline on prostate cancer screening and early diagnosis of prostate cancer can serve as a reference in terms of the management of PSA kinetics for men treated with testosterone therapy. ${ }^{75}$ While discontinuation of testosterone therapy may be advised while investigating a PSA elevation, significant increases in PSA while on testosterone therapy should not be attributed to the use of testosterone alone and should be investigated irrespective of the use or discontinuation testosterone therapy.

26. What is a reasonable timeline to begin to observe improvements in the signs and symptoms of testosterone deficiency?

Following the initiation of testosterone therapy, serum concentrations of testosterone are known to correct earlier than the symptomatic, structural and metabolic signs associated with TD. ${ }^{76,77}$ As such, patients should be counselled that symptom response will not be immediate. Expectations for treatment response should be established with each patient. Patients can anticipate improvements in many of the common symptoms of TD (libido, energy levels, sexual function) after 3 months of treatment or longer. Metabolic and structural (body composition, muscle mass, bone density) changes may take upwards of 6-months. ${ }^{77}$ In addition, patients should be counselled that diet and exercise in combination with testosterone therapy is recommended for body composition changes.

Appreciating this pattern of response to testosterone therapy is fundamental when determining the impact of treatment and the appropriate timing of follow-up evaluations while on therapy. For example, if patients undergo a symptom review and measurement of testosterone levels too early ( $<3$ months), it may lead both physicians and patients to conclude that the treatment has not been impactful (i.e. normal levels of testosterone without symptomatic/structural/metabolic benefit). However, if the same assessment was scheduled 3-6 months after the initiation of therapy, the clinical response tends to be more reflective of normalized levels of serum testosterone.

\section{How and when should testosterone therapy be discontinued?}

For most men, the initiation of testosterone therapy is discretionary and is based on the nature of their signs and symptoms, their degree of disability and impact on quality of life. Testosterone therapy should be discontinued if significant adverse events or contraindications to treatment arise or if there is no clinical improvement despite normalization of serum testosterone levels 
after an adequate therapeutic trial (approximately 3 months). ${ }^{5}$ There is no need for tapered dose titration when discontinuing of testosterone therapy (LE-high, strong recommendation).

\section{Summary}

Testosterone deficiency is common among Canadian men and is associated with significant health conditions that negatively impact quality of life. Based on a national needs-assessment of Canadian physicians, the diagnosis, management and risks associated with TD was identified as an area in need of educational tools and standard setting to enhance the quality of clinical practice. The current guideline is offered as a practical, user-friendly, evidence-based reference to support healthcare providers and ensure that men in need of testosterone therapy are diagnosed and treated effectively and safely. 


\section{References:}

Canadian Urological Association clinical practice guideline: Testosterone deficiency in men evidence-based Q\&A

Ethan D. Grober, MD1; Yonah Krakowsky, MD2; Mohit Khera, MD3; Daniel T. Holmes, MD4; Jay C. Lee, MD5; John E. Grantmyre, MD6; Premal Patel, MD7; Richard A. Bebb, MD8; Ryan Fitzpatrick, MD9; Jeffrey D. Campbell, MD10; Serge Carrier, MD11; Abraham Morgentaler, MD12

1Division of Urology, Women's College Hospital \& Sinai Health System, Department of Surgery, University of Toronto, Toronto, ON, Canada; 2Division of Urology, Women's College Hospital \& Sinai Health System, Department of Surgery, University of Toronto, Toronto, ON, Canada; 3Scott Department of Urology, Baylor College of Medicine, Houston, TX, United States; 4Department of Pathology \& Laboratory Medicine, University of British Columbia, Vancouver, BC, Canada; 5Division of Urology, Department of Surgery, University of Calgary, Calgary, AB, Canada; 6Department of Urology, Dalhousie University, Halifax, NS, Canada; 7Division of Urology, Department of Surgery, University of Manitoba, Winnipeg, MB, Canada; 8Division of Endocrinology \& Department of Urological Sciences, University of British Columbia, Vancouver, BC, Canada; 9Division of Urology, Women's College Hospital \& Sinai Health System, Department of Surgery, University of Toronto, Toronto, ON, Canada; 10Division of Urology, Department of Surgery, University of Western Ontario, London, ON, Canada 11Division of Urology, Department of Surgery, McGill University, Montreal, QC, Canada; 12Department of Urology, Harvard Medical School, Boston, MA, United States

Cite as: Grober ED, Krakowsky Y, Khera M, et al. Canadian Urological Association clinical practice guideline: Testosterone deficiency in men - evidence-based Q\&A. Can Urol Assoc J 2021 February 23; Epub ahead of print. http://dx.doi.org/10.5489/cuaj.xxxx

Published online February 23, 2021

$* * *$

Introduction

As part of their ongoing commitment to education and best practice standards, the Canadian Urological Association (CUA) solicited the creation of a clinical practice guideline dedicated to testosterone deficiency (TD) in men. 
Modern androgen therapy essentially began when testosterone was chemically synthesized in 1935.1 This scientific discovery (along with the discovery of other sex hormones - estrogen and progesterone) was awarded the Nobel Prize in Chemistry in 1939.2 Throughout most of its history, the concept of TD and replacement has been associated with confusion and controversy. Concerns relating to prostate and cardiovascular health, performance enhancement via steroid abuse and the perception that TD is simply part of the "normal" process of aging has resulted in reluctance of many healthcare providers to diagnose and treat the condition.3

In 2013, a comprehensive Canada-wide needs assessment revealed that over $25 \%$ of Canadian physicians were uncomfortable with the diagnosis and treatment of TD and identified significant knowledge gaps related to patient management. 4 To address the knowledge gaps, the Canadian Clinical Practice Guideline on the Diagnosis and Management of Testosterone Deficiency Syndrome in Adult Males was published in the CMAJ.5 As part of its mandate, the guideline was offered as a "living document," to be updated periodically in a perpetual effort to incorporate advances in scientific and clinical discovery. The current CUA guideline was approached in this spirit and serves as an educational tool that builds on existing knowledge and offers a contemporary update to the literature.

Methods

The current guideline is structured as a series of Questions \& Answers (Q\&A) relating to the diagnosis and management of TD. By way of this structure, the guideline offers a practical, evidence-based overview and recommendations related to the diagnosis, treatment, monitoring, risks and benefits of TD and testosterone therapy. It was designed to be a straightforward and user-friendly guide for specialists (urologists, endocrinologists, psychiatrists, geriatricians), general practitioners and the patients they treat. The guideline offers Canadian-specific content with respect to knowledge and attitudes related to TD, laboratory diagnostic testing, Health Canada approved treatment formulations and funding considerations.

The authorship group represents a panel of Canadian and international experts in the fields of men's health and TD. Representation is inter-provincial and multi-disciplinary covering the fields of urology, internal medicine/endocrinology and clinical biochemistry.

Relevant publications on TD and testosterone therapy were sought using a combination of Medline, EMBASE, the Cochrane Central Register of Controlled Trials (CEN $\neg$ TRAL) and PubMed searches. The search strategy involved a series of key words including: testosterone deficiency, hypogonadism, low testosterone, androgen deficiency, andropause and testosterone replacement therapy. Filters included English language, human studies and an index date up to December 1, 2020. Recently published guidelines from the Canadian Men's Health Foundation, American Urological Association, The Endocrine Society and the European Association of Urology were included as supportive, summative content.5-9

Whenever possible, guideline statements and recommendations have been assigned levels of evidence (LE) based on the GRADE Working Group framework (LE: high, moderate, low, very 
low quality of evidence).10 In brief, randomized controlled trials were initially rated as highquality and observa $\neg$ tional studies as low-quality evidence. Additionally, the strength or importance of the recom $\neg$ mendations was also graded as either "strong" or "weak" based on the quantity, quality, and consistency of the evidence available.

Following a systematic search, panel members were asked to review the identified literature and existing guidelines in an effort to highlight areas appropriate for an evidence-based review and update.11 Additionally, each member was surveyed and independently tasked to identify a series of fundamental questions relating to TD that should be addressed or updated as part of the guideline (i.e. common questions that colleagues ask of each other and patients ask of their physicians). Submissions were collated and common themes were identified. The proposed list of questions was collectively reviewed by panel members and finalized through consensus. Questions were assigned to each author based on clinical expertise for an evidence-based Answer. Responses were collectively reviewed by all panel members for feedback, clarification and consensus.

Evidenced-based Q\&A

Practical overview

1.What is the definition of testosterone deficiency?

Testosterone deficiency is a clinical and biochemical syndrome that may occur in men in association with advancing age, however younger men with certain conditions may also be affected. The condition is characterized by deficient testicular production of testosterone, with or without changes in receptor sensitivity to androgens. It may affect multiple organ systems and can result in significant health consequences and a negative impact on quality of life. 5,12 2.What is the prevalence of testosterone deficiency among Canadian men?

The prevalence of TD varies between populations and is difficult to determine in the absence of wide-spread, routine testing which is currently not the standard of care. Current studies evaluating the true prevalence of TD are conflicting due to variable reporting measures, testosterone level cut-offs and testing protocols. The crude Canadian prevalence of biochemical TD is estimated to be approximately $25 \%$ among men aged 40-62 years. 13 The rate of TD increases with age. Appreciating that prevalence rates will vary based on case definitions and biochemical testosterone thresholds, the prevalence of TD is estimated to be $4-12 \%$ in men between age $50-59$ years, $9-23 \%$ in men aged $60-69$ years, and $28-49 \%$ in men over 70 years old. $6,14,15$

3.What are the common signs and symptoms of testosterone deficiency?

Testosterone deficiency may present with a variety of clinical manifestations including sexual, cognitive and physical/structural signs and symptoms (Table 1). Common signs of TD include decreased libido and vitality, sexual dysfunction, fatigue and/or mood changes.5,16 The signs and symptoms of TD are not exclusive to this syndrome and are commonly associated with other 
conditions related to male aging. As such, the clinical presentation should be correlated with biochemical confirmation of low/equivocal serum testosterone levels. 6

4. Why does testosterone deficiency occur?

Testosterone production primarily occurs in the Leydig cells of the testis and is regulated by the hypothalamic-pituitary-gonadal-axis (HPG, Figure 1).17 For normal testosterone production, gonadotropin releasing hormone $(\mathrm{GnRH})$ is released in a pulsatile manner from the hypothalamus. GnRH stimulates the production and release of luteinizing hormone (LH) and follicle stimulating hormone (FSH) from the anterior pituitary. LH stimulates testosterone production by the Leydig cells in the testis. The HPG axis is regulated by a negative feedback loop whereby high levels of testosterone send a feedback signal (via estradiol and testosterone) to both the pituitary and the hypothalamus to inhibit ongoing testosterone production. 17 This pathway is essential for testosterone production and any disruptions can cause downstream effects and inhibit the production of testosterone.

Clinical TD results when the body fails to produce adequate levels of testosterone. If there is an intrinsic failure at the level of the testis to produce testosterone, it is termed primary hypogonadism. By contrast, if there is a hormonal/gonadotropin signaling a problem within the brain (hypothalamus or pituitary), it is considered secondary hypogonadism. Primary hypogonadism results in low levels of testosterone but high levels of gonadotropins (LH, FSH). The brain is trying to amplify its signal to the testis to produce testosterone. Secondary hypogonadism is a result of a neural signaling deficiency and clinically manifests with low levels of testosterone and low or normal levels of gonadotropins. A combined pattern of primary and secondary hypogonadism is common. TD or hypogonadism occurs as men age due to several factors including a decrease in Leydig cell size, reduced Leydig cell sensitivity to LH, increases in systemic estrogen levels and a diminution of GnRH pulsatility.18,19 Multiple medications and medical conditions are also responsible for TD (Table 2).

Diagnosis

5.How best to establish the diagnosis of testosterone deficiency with history \& physical examination?

The diagnosis of TD is established through the collective findings from history, physical examination and serum testosterone measurements (Level of Evidence (LE)-moderate, strong recommendation). The clinical manifestations of TD are non-specific with recognized variability from patient to patient. Table 1 lists clinical signs and/or symptoms commonly associated with testosterone deficiency and the medical history should be focused towards identifying the cognitive, sexual and physical/structural manifestations of TD.5-7,9 
Physical examination should be undertaken with particular attention to the following testosterone dependent tissues and organs.

- $\quad$ Testicles (size, firmness, location)

- $\quad$ Penile development (length, location of urethral opening)

- $\quad$ Prostate size and consistency by performing a digital rectal examination (DRE)

- $\quad$ Secondary sex characteristics: facial and body hair, changes with puberty

- $\quad$ Breast tissue development (gynecomastia)

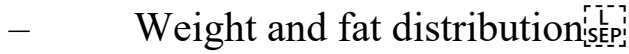

- $\quad$ Muscle mass and bone structure/function

6.Are there valid screening questionnaires for testosterone deficiency? Are they helpful in establishing the diagnosis?

Several validated screening questionnaires for TD exist including the Androgen Deficiency in the Aging Male (ADAM), Quantitative ADAM, Aging Males' Symptoms Scale, Massachusetts Male Aging Study Questionnaire as well as questionnaires that focus on a specific common element of testosterone deficiency (i.e. erectile dysfunction): International Index of Erectile Function, Sexual Health Inventory for Men and Erection Hardness Score.13,20-24

Due to their lack of specificity (approximately 20\%), screening questionnaires alone should not be substituted for a detailed history, physical examination and biochemical testing when establishing the diagnosis of TD.6,9,25 Standardized questionnaires are often helpful for academic/research purposes and can act as an initial screen to facilitate discussion and highlight areas of focus for further evaluation (LE-low, weak recommendation).

7.What is the recommended laboratory test to diagnose testosterone deficiency?

Challenges have always existed in the quality of commercial immunoassays to accurately measure testosterone relative to validated reference methodologies - the gold standards being equilibrium dialysis and mass spectrometry.26 The development of the Centre for Disease Controls Hormone Standardization (HoSt) program (https://www.cdc.gov/labstandards/hs_standardization.html) has forced many commercial immunoassay vendors to improve their calibration and accuracy in the measurement of testosterone.27 Total testosterone (morning draw: 7am-11am) remains the best initial screening test to diagnose TD.5,6 Calculated free or bioavailable testosterone based on age-dependant standards of total testosterone, sex hormone binding globulin (SHBG) and albumin concentrations can be determined to resolve equivocal total testosterone measures in symptomatic men.5(LE-moderate, strong recommendation)

8. What is the biochemical level or cut off to diagnose testosterone deficiency? The clinical diagnosis of TD relies on the correlation of recognized signs and symptoms with biochemical confirmation of deficient serum testosterone concentrations. Establishing an absolute biochemical cut-off diagnostic for TD is challenging due to several factors including: limitations in measurement accuracy; laboratory and immunoassay variability; lack of age- 
related reference standards; unknown baseline testosterone levels in individual patients; and variable target organ receptor sensitivity to circulating testosterone concentrations. Most clinician experts and previously published guidelines would suggest that a total testosterone $<10$ $\mathrm{nmol} / \mathrm{L}$ represents a reasonable diagnostic threshold consistent for TD, while appreciating that some patients may manifest symptoms of TD at higher levels.5,6,12 Testosterone measures can be used as a complimentary tool (as opposed to an absolute or diagnostic one), to support the diagnosis of TD in the context of characteristic signs and symptoms identified by way of a detailed clinical evaluation (LE-low, weak recommendation).

9.Besides measuring testosterone, what adjunctive laboratory testing is indicated? In patients with established TD, clinicians should measure serum luteinizing hormone levels (LH) to help classify the testosterone deficiency as primary (high LH) or secondary (low/normal LH).5,6,12

Serum prolactin levels should be measured in patients suspected of having secondary testosterone deficiency (low testosterone levels combined with low or low/normal LH levels). Patients with persistently high prolactin levels of unknown etiology should undergo evaluation for endocrine disorders and to rule out a prolactinoma. 6

Serum estradiol should be measured in testosterone deficient patients who present with breast symptoms or gynecomastia prior to the commencement of testosterone therapy.6

(LE-moderate, strong recommendation)

10. What are potentially reversible causes of testosterone deficiency that physicians should consider?

Prior to initiating testosterone therapy, physicians should consider and manage any potentially reversible causes of TD5 (LE-moderate, strong recommendation). These may include:

- $\quad$ Acute illness

- Obesity

- $\quad$ Medication use (i.e. opioids, glucocorticoids)

- $\quad$ Extreme nutritional deficiencies and anorexia

- $\quad$ Extreme exercise

- $\quad$ Sleep Apnea

- Hyperprolactinemia

- Hemochromatosis

- Hypothalamic and pituitary disorders (surgery, trauma, radiation, infiltrative disorders)

Following treatment or stabilization of these conditions, reassessment of serum testosterone levels and the signs and symptoms of TD is recommended.

11. What are the common co-morbid conditions associated with testosterone deficiency? 
Testosterone deficiency is associated with several specific health conditions that may prompt investigation and management of serum testosterone levels to validate such associations and compliment the management of such conditions.5,7

- $\quad$ Type 2 diabetes \& insulin resistance

- $\quad$ Metabolic syndrome

- Obesity

- Osteopenia/osteoporosis

- COPD

- $\quad$ Sleep Apnea

- $\quad$ HIV/AIDS

Treatment

12.What are the goals and benefits of testosterone therapy?

The primary goals of testosterone therapy are to mitigate the negative signs and symptoms of testosterone deficiency and improve quality of life while achieving eugonadal (normal) levels of testosterone. 5

The benefits of treatment will vary according to the symptom complex of each patient. The Testosterone Trials - the largest placebo-controlled, double-blinded, multicenter, randomized controlled trial of testosterone therapy in symptomatic hypogonadal older men - demonstrated significant improvements in sexual activity, sexual desire, erectile function, mood and depressive symptoms, anemia and bone mineral density among men receiving testosterone therapy.28,29 13. What are the current treatment options for testosterone deficiency in Canada? Table 3 summarizes the testosterone treatment formulations currently approved by Health Canada.

Compounded testosterone products are available at many compounding and online pharmacies within Canada, however published data has demonstrated significant variability of testosterone concentrations within such products leading to concerns regarding efficacy and safety. 30

The choice testosterone therapy and route of administration should be a topic of discus $\neg$ sion between the physician and the patient using a shared decision-making approach. Factors influencing this choice include safety, efficacy, tolerabil $\neg$ ity, availability, preference and cost/insurance coverage.

14. What is the recommended approach to treating a patient with characteristic symptoms of testosterone deficiency with a "normal" testosterone level?

Patients may present with a clinical picture consistent with TD, yet serum testosterone concentrations within the "normal" reference range. Efforts should be made to rule out conditions with an overlapping symptom complex (i.e. depression, hypothyroidism, sleep disorders). 
Challenges in the measurement of serum testosterone, inconsistent normal reference ranges between laboratories, the lack of a "baseline" reference level of testosterone earlier in a man's life, variable androgen receptor sensitivity to a given concentration of testosterone and the ability of elevated sex-hormone binding globulin (SHBG) levels to effectively neutralize circulating testosterone may account for a symptomatic presentation with normal total serum testosterone levels. In equivocal cases, measurement of SHBG and calculation of free and/or bioavailable testosterone may provide additional insight upon which to better interpret testosterone concentrations and rationalize treatment.7,27,31

Recognizing such limitations, a supervised trial of testosterone therapy (3-months) with close monitoring of both the symptomatic and biochemical response to treatment is recommended.5 (LE-low, weak recommendation)

15. What is the recommended approach to treating a patient with NO symptoms of testosterone deficiency but a "low" testosterone level?

In general, hypogonadal men who are asymptomatic with no impact on quality of life should not be offered testosterone therapy. Investigation and consideration of treatment should be initiated in men with unexplained anemia or sarcopenia, chronic use of glucocorticoid or opioid therapy, as well as patients with human immunodeficiency virus (HIV) experiencing weight loss, irrespective of the presence of other symptoms of TD.5,7 (LE-moderate, weak recommendation) 16. What is the likelihood that my patient will respond/benefit from treatment? Clinical response rates may vary according to several factors including baseline level of testosterone, the choice of testosterone therapy, dosage and delivery method as well as the nature and severity of each individual patient's symptoms. Overall, testosterone therapy has been shown to effectively normalize serum testosterone concentrations in the majority $(>90 \%)$ of treated men.29,32 Due to the varied clinical spectrum associated with TD and the limitations associated with objective measurement of symptoms, at the present time there is insufficient data to specifically quantify what proportion of hypogonadal men overall will respond symptomatically to treatment, and if any specific characteristics make a patient more likely to benefit. 6 As such, practitioners are encouraged to concurrently monitor both patient-specific symptomatic responses from baseline as well as changes in serum testosterone levels while on treatment. Patients with a favorable biochemical and symptomatic response to treatment should continue with therapy. An alternative diagnosis should be considered in patients who fail to respond symptomatically despite normalization of testosterone levels and an adequate trial of therapy - approximately 3 months. (LE-low, weak recommendation)

17. What is the best treatment approach to a patient with testosterone deficiency who is interested in fertility preservation?

Many patients and clinicians are unaware that exogenous testosterone therapy can serve as a contraceptive and result in depressed sperm production leading to male infertility.33,34 A recent survey of urologists found that roughly $25 \%$ would treat infertile males with testosterone while 
they pursued a pregnancy.34 Exogenous testosterone administration results in negative feedback (mainly via testosterone converted to estradiol) to the pituitary leading to suppression of the primary signals for sperm (FSH) and testosterone $(\mathrm{LH})$ production at the testicular level (Figure 1).17 Hypogonadal patients who are interested in fertility preservation should be cautioned against the isolated use of exogenous testosterone and instead pursue treatments that increase endogenous serum testosterone production (LE-high, strong recommendation). Human chorionic gonadotropin (HCG), selective estrogen receptor modulators (SERMs - clomiphene, tamoxifen) and aromatase inhibitors (AIs - anastrozole, letrozole, testolactone) are alternative treatment options (off-label) to increase endogenous serum testosterone levels without threatening fertility potential.5,6,33,35,36

As a unique short-acting formulation, testosterone nasal gel (Natesto) may result in less suppression of gonadotropins (LH/FSH) and semen parameters and may represent a treatment option for symptomatic hypogonadal men who desire to maintain fertility potential.37,38 Consultation with a male fertility specialist is recommended.

18. What is the suggested level of testosterone to achieve while on treatment?

Treating physicians should aim to prescribe the minimal required dosing to improve serum testosterone and hypogonadal symptoms. Recognizing the variations in normal ranges among the various laboratories throughout Canada, the current recommendations are to target correction of serum levels to the mid-normal reference range (total testosterone 14-17 nmol/L, LE-low, weak recommendation).5,39. Patients without significant symptomatic improvement and serum testosterone levels measured below the mid-normal range can be considered for dose escalation to improve clinical efficacy. Clinical experience supports raising testosterone into the upper third of the normal range for patients who do not respond symptomatically at lower levels.6 Patients with satisfactory resolution of their symptoms, but serum testosterone below the recommended target range typically do not require dose adjustment.28 Dose reductions should be prescribed in patients with serum testosterone concentrations consistently measuring above the normal range while on treatment.

19. What is the evidence for herbal or natural testosterone "boosters" in treating testosterone deficiency?

Current evidence-based reviews of the most common ingredients in testosterone-boosters (Tboosters) on the market have found minimal to no evidence demonstrating their efficacy.40 Many of the ingredients within such compounds have never been tested for safety and/or efficacy in human trials. Among human trials, only 30\% demonstrated an improvement in testosterone levels, $2 \%$ resulted in a decrease in testosterone and $68 \%$ had either an indeterminant effect or no effect at all on testosterone levels. 41 Concerningly, several case studies have demonstrated severe adverse events from T-boosters that contain banned/illicit substances including steroids.42,43 Currently, T-boosters cannot be recommended by health practitioners and patients 
should be cautioned regarding the unknown efficacy and safety of T-boosters along with the possibility for severe adverse events. Regulated, evidence-based treatments should be offered as an alternative in symptomatic hypogonadal men (LE-moderate, strong recommendation).

20. Aside from testosterone, are there other treatments that can be used to treat testosterone deficiency?

Similar to other chronic conditions, lifestyle modification may have a role in managing TD (LElow, weak recommendation). A bidirectional relationship between obesity and testosterone levels has been demonstrated. Mechanistically, obesity lowers testosterone levels due to reductions in LH release and greater aromatization of testosterone to estradiol within adipose tissue.44,45 Weight loss, dietary restriction and bariatric surgery all have been shown to significantly increase serum testosterone levels.46,47 Exercise, including resistance training, has been shown to result in relatively modest increases in serum testosterone levels.48,49 Finally, sleep patterns have shown to influence testosterone concentrations with persistent sleep deprivation/restriction lowering testosterone levels by approximately 10-15\%.50,51 A commitment to structured sleep program may negate the impact of limited sleep on testosterone concentrations. Some selective symptoms of TD can be managed with symptom specific treatments (i.e. PDE5 inhibitors for erectile dysfunction). 52

Treatment risks

21.Does testosterone replacement therapy increase the risk of prostate cancer?

Despite historical teachings about the relationship between testosterone on prostate cancer biology and risk, there is now consistent evidence that testosterone therapy does not increase a man's risk for developing prostate cancer. In the largest randomized controlled trial (RCT) to date (The Testosterone Trials), involving 790 men 65 years and older treated for 12-months with either testosterone gel or placebo, despite close surveillance, only one man developed prostate cancer during the course of the study.29 Supporting such observations, a meta-analysis of 22 RCTs involving 2351 men revealed no greater risk of developing prostate cancer among those who received testosterone therapy compared with those who received placebo.53 There is no association between the risk prostate cancer and serum testosterone concentrations. Men with the highest naturally occurring (endogenous) testosterone levels, do not exhibit significantly higher prostate specific antigen (PSA) levels or a greater subsequent risk developing prostate cancer than men with the lowest levels of endogenous testosterone.54,55 Finally, the evolving literature suggests among appropriately-selected hypogonadal men with prostate cancer managed with active surveillance, radical prostatectomy or radiation therapy, testosterone therapy does not appear to significantly increase the risk of biochemical (PSA) progression, local or metastatic progression, overall mortality and cancer-specific mortality.56-58

Symptomatic men with TD who have been diagnosed with localized prostate cancer and treated (surgery, radiation) or followed with active surveillance without evidence of active disease can considered for a medically supervised trial of testosterone therapy (LE-low, weak 
recommendation). Consultation with a urologic specialist is recommended. Patients with metastatic or high-risk prostate cancer who are likely to require androgen deprivation therapy should not be offered testosterone therapy (LE-moderate, strong recommendation). 5

22.Does testosterone replacement therapy increase the risk of benign prostatic hyperplasia (BPH) progression and lower urinary tract symptom (LUTS)?

A collective review of the contemporary evidence to date suggests that testosterone therapy does not increase the risk of urinary symptoms due to BPH.59-61 Consistent with previous metaanalyses, in the Testosterone Trials, the largest RCT to date, involving 790 men aged 65 years and older, a similar rate of worsening urinary symptoms, suggestive of $\mathrm{BPH}$, were noted over the 1-year study period for those who received testosterone therapy compared with those who received placebo.29 Moreover, in a review of 16 RTCs involving 1030 men, testosterone therapy had no significant impact on prostate volume.61 Several studies have demonstrated improvement in urinary symptoms, peak urinary flow rates and voided volumes with the initiation of testosterone treatment in hypogonadal men which is theoretically related to its impact on muscular (bladder detrusor) function.59,60,62,63

While individual responses to any treatment, including testosterone vary, evidence suggest that testosterone therapy does not generally increase the risk of worsening voiding symptoms. As such, testosterone therapy can safely be administered to men with BPH and lower urinary tract symptoms (LE-moderate, weak recommendation).

23.Does testosterone replacement therapy increase the risk of cardiovascular disease? Evidence suggests that low endogenous (natural) levels of testosterone are associated with increased cardiovascular risk including higher rates of obesity, diabetes, dyslipidemia, metabolic syndrome and demonstrates an increased risk of cardiovascular death.64-66

The published literature on the impact of testosterone therapy on cardiovascular risk is conflicting and continues to evolve. Prior to 2010, the majority of studies and large metaanalyses suggests cardiovascular benefits among hypogonadal men initiated on testosterone therapy including: improved lean muscle mass, deceased total body fat and waist circumference, improved glycemic control (hemoglobin A1C levels), decreased cardiovascular events and decreased mortality.67-69

Following 2010, a series of high-impact publications raised concerns regarding the use of testosterone therapy and an increased risk cardiovascular events including myocardial infarction, stroke and death.70,71 Despite significant methodological concerns with study design and reporting, in 2014 the Food and Drug Administration (FDA) and Health Canada released warnings regarding the use of testosterone therapy and the possible risk of cardiovascular events.72,73 These warning garnered significant media and medical attention.

To date, there are no large, long-term placebo-controlled trials to help make definitive statements on testosterone therapy and cardiovascular risk. The literature available suggests that untreated testosterone deficient men are at increased risk of heart disease, cardiovascular events 
and death.64-66 Based on the best available evidence, symptomatic hypogonadal men with stable cardiovascular disease remain candidates for a medically supervised trial of testosterone therapy.5 An individualized risk-benefit assessment is prudent (LE-low, weak recommendation).

24. What are the contraindications to testosterone therapy?

Contraindications to testosterone therapy include:5-8,11

- $\quad$ Allergy or hypersensitivity to prescribed treatment

- $\quad$ Known or suspected male breast cancer

- $\quad$ Patients who desire fertility preservation (refer to Question and Answer \#17)

- $\quad$ Patients with metastatic or high-risk prostate cancer who are likely to require androgen deprivation therapy

- $\quad$ Patients with unstable cardiovascular disease

Monitoring

25.What monitoring is required for a patient receiving testosterone therapy?

After initiating testosterone therapy, patients should have regular monitoring for response to treatment and adverse events. Table 4 summarizes an evidence-based monitoring schedule for patients on testosterone therapy. 5-7,9

Adjustments to treatment dose and delivery can be made based on patient response, adverse events, and bloodwork results. Testosterone levels (+/- gonadotropins) should be measured to determine adherence and absorption of treatment (LE-moderate, strong recommendation).5,6 The recommended timing of testosterone measurement relative to the last treatment dose will vary depending on the testosterone formulation (i.e. mid-cycle for injectables) and patients should be counselled appropriately to ensure accurate interpretation of results and response to treatment.

A patient's hematocrit should be followed to ensure they are not developing polycythemia. Polycythemia is a common side effect of testosterone therapy and may be associated with thromboembolic events (LE-moderate, strong recommendation).69,74 Management strategies for persistent treatment induced polycythemia (hematocrit levels 55\% or greater) may include a dose reduction of testosterone (concentration or application frequency), drug holiday, formulation change or blood donation/phlebotomy.

Prostate specific antigen (PSA) testing and digital rectal examination should be performed to monitor prostate health in accordance with the evidence-based guidelines for prostate cancer screening (LE-low, weak recommendation).75 The Canadian Urological Association guideline on prostate cancer screening and early diagnosis of prostate cancer can serve as a reference in terms of the management of PSA kinetics for men treated with testosterone therapy.75 While discontinuation of testosterone therapy may be advised while investigating a PSA elevation, significant increases in PSA while on testosterone therapy should not be attributed to the use of 
testosterone alone and should be investigated irrespective of the use or discontinuation testosterone therapy.

26. What is a reasonable timeline to begin to observe improvements in the signs and symptoms of testosterone deficiency?

Following the initiation of testosterone therapy, serum concentrations of testosterone are known to correct earlier than the symptomatic, structural and metabolic signs associated with TD.76,77 As such, patients should be counselled that symptom response will not be immediate. Expectations for treatment response should be established with each patient. Patients can anticipate improvements in many of the common symptoms of TD (libido, energy levels, sexual function) after 3 months of treatment or longer. Metabolic and structural (body composition, muscle mass, bone density) changes may take upwards of 6-months.77 In addition, patients should be counselled that diet and exercise in combination with testosterone therapy is recommended for body composition changes.

Appreciating this pattern of response to testosterone therapy is fundamental when determining the impact of treatment and the appropriate timing of follow-up evaluations while on therapy. For example, if patients undergo a symptom review and measurement of testosterone levels too early ( $<3$ months), it may lead both physicians and patients to conclude that the treatment has not been impactful (i.e. normal levels of testosterone without symptomatic/structural/metabolic benefit). However, if the same assessment was scheduled 3-6 months after the initiation of therapy, the clinical response tends to be more reflective of normalized levels of serum testosterone.

27.How and when should testosterone therapy be discontinued?

For most men, the initiation of testosterone therapy is discretionary and is based on the nature of their signs and symptoms, their degree of disability and impact on quality of life. Testosterone therapy should be discontinued if significant adverse events or contraindications to treatment arise or if there is no clinical improvement despite normalization of serum testosterone levels after an adequate therapeutic trial (approximately 3 months). 5 There is no need for tapered dose titration when discontinuing of testosterone therapy (LE-high, strong recommendation). Summary

Testosterone deficiency is common among Canadian men and is associated with significant health conditions that negatively impact quality of life. Based on a national needs-assessment of Canadian physicians, the diagnosis, management and risks associated with TD was identified as an area in need of educational tools and standard setting to enhance the quality of clinical practice. The current guideline is offered as a practical, user-friendly, evidence-based reference to support healthcare providers and ensure that men in need of testosterone therapy are diagnosed and treated effectively and safely. 


\section{References}

1. Nieschlag E, Nieschlag S. The history of discovery, synthesis and development of testosterone for clinical use. European Journal of Endocrinology. Published online 2019. doi:10.1530/EJE-19-0071

2. Morales A. The Long and Tortuous History of the Discovery of Testosterone and Its Clinical Application. Journal of Sexual Medicine. Published online 2013. doi:10.1111/jsm.12081

3. Grober ED. Testosterone deficiency and replacement: Myths and realities. Journal of the Canadian Urological Association. Published online 2014. doi:10.5489/cuaj.2309

4. Canadian Men's Health Foundation Multidisciplinary Guidelines Task Force on TDS. Data on File. 2013.; 2013.

5. Morales A, Bebb RA, Manjoo P, et al. Diagnosis and management of testosterone deficiency syndrome in men: clinical practice guideline. Canadian Medical Association Journal. 2015;187(18). doi:10.1503/cmaj.150033

6. Mulhall JP, Trost LW, Brannigan RE, et al. Evaluation and Management of Testosterone Deficiency: AUA Guideline. Journal of Urology. Published online 2018. doi:10.1016/j.juro.2018.03.115

7. Bhasin S, Brito JP, Cunningham GR, et al. Testosterone Therapy in Men with Hypogonadism: An Endocrine Society. Journal of Clinical Endocrinology and Metabolism. Published online 2018. doi:10.1210/jc.2018-00229

8. Bhasin S, Cunningham GR, Hayes FJ, et al. Testosterone therapy in men with androgen deficiency syndromes: An endocrine society clinical practice guideline. Journal of Clinical Endocrinology and Metabolism. Published online 2010. doi:10.1210/jc.2009-2354

9. Dohle G, Arver S, Bettocchi C, Jones TH, Kliesch S, Punab M. Guidelines on Male Hypogonadism. European Association of Urology. Published online 2015.

10. Schünemann H, Brożek J, Guyatt G, Oxman A. GRADE Handbook for Grading Quality of Evidence and Strength of Recommendations.; 2013.

11. Kwong JCC, Krakowsky Y, Grober E. Testosterone Deficiency: A Review and Comparison of Current Guidelines. Journal of Sexual Medicine. Published online 2019. doi:10.1016/j.jsxm.2019.03.262

12. Morales A, Bella AJ, Chun S, et al. A practical guide to diagnosis, management and treatment of testosterone deficiency for Canadian physicians. Journal of the Canadian Urological Association. Published online 2010. doi:10.5489/cuaj.880

13. Morley JE, Charlton E, Patrick $\mathrm{P}$, et al. Validation of a screening questionnaire for androgen deficiency in aging males. Metabolism: Clinical and Experimental. Published online 2000. doi:10.1053/meta.2000.8625 
14. Araujo AB, Esche GR, Kupelian V, et al. Prevalence of symptomatic androgen deficiency in men. Journal of Clinical Endocrinology and Metabolism. Published online 2007. doi:10.1210/jc.2007-1245

15. Harman SM, Metter EJ, Tobin JD, Pearson J, Blackman MR. Longitudinal effects of aging on serum total and free testosterone levels in healthy men. Journal of Clinical Endocrinology and Metabolism. Published online 2001. doi:10.1210/jcem.86.2.7219

16. Wu FCW, Tajar A, Beynon JM, et al. Identification of Late-Onset Hypogonadism in Middle-Aged and Elderly Men. New England Journal of Medicine. Published online 2010. doi:10.1056/nejmoa0911101

17. Corradi PF, Corradi RB, Greene LW. Physiology of the Hypothalamic Pituitary Gonadal Axis in the Male. Urologic Clinics of North America. Published online 2016. doi:10.1016/j.ucl.2016.01.001

18. Golan R, Scovell JM, Ramasamy R. Age-related testosterone decline is due to waning of both testicular and hypothalamic-pituitary function. Aging Male. Published online 2015. doi:10.3109/13685538.2015.1052392

19. Rubens R, Dhont M, Vermeulen A. Further studies on leydig cell function in old age. Journal of Clinical Endocrinology and Metabolism. Published online 1974. doi:10.1210/jcem-39-1-40

20. Mohamed O, Freundlich RE, Dakik HK, et al. The quantitative ADAM questionnaire: A new tool in quantifying the severity of hypogonadism. International Journal of Impotence Research. Published online 2010. doi:10.1038/ijir.2009.35

21. Heinemann LAJ, Zimmermann T, Vermeulen A, Thiel C, Hummel W. A new "aging males" symptoms' rating scale. Aging Male. Published online 1999. doi:10.3109/13685539909003173

22. Feldman HA, Goldstein I, Hatzichristou DG, Krane RJ, McKinlay JB. Impotence and its medical and psychosocial correlates: Results of the Massachusetts Male Aging Study. Journal of Urology. Published online 1994. doi:10.1016/S00225347(17)34871-1

23. Mulhall JP, Goldstein I, Bushmakin AG, Cappelleri JC, Hvidsten K. Validation of the erection hardness score. Journal of Sexual Medicine. Published online 2007. doi:10.1111/j.1743-6109.2007.00600.x

24. Rosen RC, Riley A, Wagner G, Osterloh IH, Kirkpatrick J, Mishra A. The international index of erectile function (IIEF): A multidimensional scale for assessment of erectile dysfunction. Urology. Published online 1997. doi:10.1016/S0090-4295(97)00238-0

25. Tancredi A, Reginster JY, Schleich F, et al. Interest of the Androgen Deficiency in Aging Males (ADAM) questionnaire for the identification of hypogonadism in 
elderly community-dwelling male volunteers. European Journal of Endocrinology. Published online 2004. doi:10.1530/eje.0.1510355

26. Fiers T, Wu F, Moghetti P, Vanderschueren D, Lapauw B, Kaufman JM. Reassessing Free-Testosterone Calculation by Liquid Chromatography-Tandem Mass Spectrometry Direct Equilibrium Dialysis. Journal of Clinical Endocrinology and Metabolism. Published online 2018. doi:10.1210/jc.2017-02360

27. Vesper HW, Botelho JC. Standardization of testosterone measurements in humans. Journal of Steroid Biochemistry and Molecular Biology. Published online 2010. doi:10.1016/j.jsbmb.2010.03.032

28. Snyder PJ, Ellenberg SS, Cunningham GR, et al. The Testosterone Trials: Seven coordinated trials of testosterone treatment in elderly men. Clinical Trials. Published online 2014. doi:10.1177/1740774514524032

29. Snyder PJ, Bhasin S, Cunningham GR, et al. Effects of Testosterone Treatment in Older Men. New England Journal of Medicine. Published online 2016. doi:10.1056/nejmoa1506119

30. Grober ED, Garbens A, Božović A, Kulasingam V, Fanipour M, Diamandis EP. Accuracy of Testosterone Concentrations in Compounded Testosterone Products. Journal of Sexual Medicine. Published online 2015. doi:10.1111/jsm.12898

31. Krakowsky Y, Conners W, Morgentaler A. Serum Concentrations of Sex Hormonebinding Globulin Vary Widely in Younger and Older Men: Clinical Data from a Men's Health Practice. European Urology Focus. Published online 2019. doi:10.1016/j.euf.2017.05.007

32. Reyes-Vallejo L, Lazarou S, Morgentaler A. Subjective sexual response to testosterone replacement therapy based on initial serum levels of total testosterone. Journal of Sexual Medicine. Published online 2007. doi:10.1111/j.17436109.2006.00381.x

33. Crosnoe LE, Grober E, Ohl D, Kim ED. Exogenous testosterone: A preventable cause of male infertility. Translational Andrology and Urology. Published online 2013. doi:10.3978/j.issn.2223-4683.2013.06.01

34. Samplaski MK, Loai Y, Wong K, Lo KC, Grober ED, Jarvi KA. Testosterone use in the male infertility population: Prescribing patterns and effects on semen and hormonal parameters. Fertility and Sterility. Published online 2014. doi:10.1016/j.fertnstert.2013.09.003

35. Coviello AD, Matsumoto AM, Bremner WJ, et al. Low-dose human chorionic gonadotropin maintains intratesticular testosterone in normal men with testosteroneinduced gonadotropin suppression. Journal of Clinical Endocrinology and Metabolism. Published online 2005. doi:10.1210/jc.2004-0802 
36. Schlegel PN. Aromatase inhibitors for male infertility. Fertility and Sterility. Published online 2012. doi:10.1016/j.fertnstert.2012.10.023

37. Gronski MA, Grober ED, Gottesman IS, Ormsby RW, Bryson N. Efficacy of Nasal Testosterone Gel (Natesto $\left.{ }^{\circledR}\right)$ Stratified by Baseline Endogenous Testosterone Levels. Journal of the Endocrine Society. Published online 2019. doi:10.1210/js.2019-00183

38. Ramasamy R, Masterson TA, Best JC, et al. Effect of Natesto on Reproductive Hormones, Semen Parameters and Hypogonadal Symptoms: A Single Center, Open Label, Single Arm Trial. The Journal of urology. Published online 2020. doi:10.1097/JU.0000000000001078

39. Corona G, Rastrelli G, Maggi M. Diagnosis and treatment of late-onset hypogonadism: Systematic review and meta-analysis of TRT outcomes. Best Practice and Research: Clinical Endocrinology and Metabolism. Published online 2013. doi:10.1016/j.beem.2013.05.002

40. Clemesha CG, Thaker H, Samplaski MK. 'Testosterone boosting' supplements composition and claims are not supported by the academic literature. World Journal of Men's Health. Published online 2020. doi:10.5534/wjmh.190043

41. Balasubramanian A, Thirumavalavan N, Srivatsav A, Yu J, Lipshultz LI, Pastuszak AW. Testosterone Imposters: An Analysis of Popular Online Testosterone Boosting Supplements. Journal of Sexual Medicine. Published online 2019. doi:10.1016/j.jsxm.2018.12.008

42. Pokrywka A, Obmiński Z, Malczewska-Lenczowska J, Fijałek Z, Turek-Lepa E, Grucza R. Insights into supplements with tribulus terrestris used by athletes. Journal of Human Kinetics. Published online 2014. doi:10.2478/hukin-2014-0037

43. Almaiman AA. Effect of testosterone boosters on body functions: Case report. Int J Heal Sciences 2018; 12:83-7. 2018;12:83-87.

44. Lima N, Cavaliere H, Knobel M, Halpern A, Medeiros-Neto G. Decreased androgen levels in massively obese men may be associated with impaired function of the gonadostat. International Journal of Obesity. Published online 2000. doi:10.1038/sj.ijo.0801406

45. Pastuszak AW, Mittakanti H, Liu JS, Gomez L, Lipshultz LI, Khera M. Pharmacokinetic evaluation and dosing of subcutaneous testosterone pellets. Journal of Andrology. Published online 2012. doi:10.2164/jandrol.111.016295

46. Corona G, Rastrelli G, Monami M, et al. Body weight loss reverts obesity-associated hypogonadotropic hypogonadism: A systematic review and meta-analysis. European Journal of Endocrinology. Published online 2013. doi:10.1530/EJE-12-0955

47. Hammoud A, Gibson M, Hunt SC, et al. Effect of roux-en-Y gastric bypass surgery on the sex steroids and quality of life in obese men. Journal of Clinical Endocrinology and Metabolism. Published online 2009. doi:10.1210/jc.2008-1598 
48. Khoo J, Tian HH, Tan B, et al. Comparing effects of low- and high-volume moderateintensity exercise on sexual function and testosterone in obese men. Journal of Sexual Medicine. Published online 2013. doi:10.1111/jsm.12154

49. Ahtiainen JP, Pakarinen A, Kraemer WJ, Häkkinen K. Acute hormonal responses to heavy resistance exercise in strength athletes versus nonathletes. Canadian Journal of Applied Physiology. Published online 2004. doi:10.1139/h04-034

50. Leproult R, van Cauter E. Effect of 1 week of sleep restriction on testosterone levels in young healthy men. JAMA - Journal of the American Medical Association.

Published online 2011. doi:10.1001/jama.2011.710

51. Cignarelli A, Castellana M, Castellana G, et al. Effects of CPAP on Testosterone Levels in Patients With Obstructive Sleep Apnea: A Meta-Analysis Study. Frontiers in Endocrinology. Published online 2019. doi:10.3389/fendo.2019.00551

52. Burnett AL, Nehra A, Breau RH, et al. Erectile Dysfunction: AUA Guideline. Journal of Urology. Published online 2018. doi:10.1016/j.juro.2018.05.004

53. Cui Y, Zong H, Yan H, Zhang Y. The effect of testosterone replacement therapy on prostate cancer: A systematic review and meta-analysis. Prostate Cancer and Prostatic Diseases. Published online 2014. doi:10.1038/pcan.2013.60

54. Roddam AW, Allen NE, Appleby P, Key TJ. Endogenous sex hormones and prostate cancer: A collaborative analysis of 18 prospective studies. Journal of the National Cancer Institute. Published online 2008. doi:10.1093/jnci/djm323

55. Grober ED, Lamb DJ, Khera M, Murthy L, Lipshultz LI. Correlation between simultaneous PSA and serum testosterone concentrations among eugonadal, untreated hypogonadal and hypogonadal men receiving testosterone replacement therapy. International Journal of Impotence Research. Published online 2008. doi:10.1038/ijir.2008.40

56. Pastuszak AW, Khanna A, Badhiwala N, et al. Testosterone Therapy after Radiation Therapy for Low, Intermediate and High Risk Prostate Cancer. Journal of Urology. Published online 2015. doi:10.1016/j.juro.2015.05.084

57. Khera M, Grober ED, Najari B, et al. Testosterone replacement therapy following radical prostatectomy. Journal of Sexual Medicine. Published online 2009. doi:10.1111/j.1743-6109.2009.01161.x

58. Morgentaler A, Lipshultz LI, Bennett R, Sweeney M, Avila D, Khera M. Testosterone therapy in men with untreated prostate cancer. Journal of Urology. Published online 2011. doi:10.1016/j.juro.2010.11.084

59. Kalinchenko S, Vishnevskiy EL, Koval AN, Mskhalaya GJ, Saad F. Beneficial effects of testosterone administration on symptoms of the lower urinary tract in men with late-onset hypogonadism: A pilot study. Aging Male. Published online 2008. doi:10.1080/13685530801953994 
60. Pearl JA, Berhanu D, François N, et al. Testosterone supplementation does not worsen lower urinary tract symptoms. Journal of Urology. Published online 2013. doi:10.1016/j.juro.2013.05.111

61. Cui Y, Zhang Y. The effect of androgen-replacement therapy on prostate growth: A systematic review and meta-analysis. European Urology. Published online 2013. doi:10.1016/j.eururo.2013.03.042

62. Shigehara K, Sugimoto K, Konaka H, et al. Androgen replacement therapy contributes to improving lower urinary tract symptoms in patients with hypogonadism and benign prostate hypertrophy: A randomised controlled study. Aging Male. Published online 2011. doi:10.3109/13685538.2010.518178

63. Haider KS, Haider A, Doros G, Traish A. Long-Term Testosterone Therapy Improves Urinary and Sexual Function, and Quality of Life in Men with Hypogonadism: Results from a Propensity Matched Subgroup of a Controlled Registry Study. Journal of Urology. Published online 2018. doi:10.1016/j.juro.2017.07.039

64. Traish AM, Saad F, Guay A. The dark side of testosterone deficiency: II. type 2 diabetes and insulin resistance. Journal of Andrology. Published online 2009. doi:10.2164/jandrol.108.005751

65. Tajar A, Huhtaniemi IT, O'Neill TW, et al. Characteristics of androgen deficiency in Late-onset hypogonadism: Results from the European Male Aging study (emas). Journal of Clinical Endocrinology and Metabolism. Published online 2012. doi:10.1210/jc.2011-2513

66. Mulligan T, Frick MF, Zuraw QC, Stemhagen A, McWhirter C. Prevalence of hypogonadism in males aged at least 45 years: The HIM study. International Journal of Clinical Practice. Published online 2006. doi:10.1111/j.1742-1241.2006.00992.x

67. Shores MM, Smith NL, Forsberg CW, Anawalt BD, Matsumoto AM. Testosterone treatment and mortality in men with low testosterone levels. Journal of Clinical Endocrinology and Metabolism. Published online 2012. doi:10.1210/jc.2011-2591

68. Haddad RM, Kennedy CC, Caples SM, et al. Testosterone and cardiovascular risk in men: A systematic review and meta-analysis of randomized placebo-controlled trials. Mayo Clinic Proceedings. Published online 2007. doi:10.4065/82.1.29

69. Fernández-Balsells MM, Murad MH, Lane M, et al. Adverse effects of testosterone therapy in adult men: A systematic review and meta-analysis. Journal of Clinical Endocrinology and Metabolism. Published online 2010. doi:10.1210/jc.2009-2575

70. Basaria S, Coviello AD, Travison TG, et al. Adverse Events Associated with Testosterone Administration. New England Journal of Medicine. Published online 2010. doi:10.1056/nejmoa1000485

71. Vigen R, O'Donnell CI, Barón AE, et al. Association of testosterone therapy with mortality, myocardial infarction, and stroke in men with low testosterone levels. 
JAMA - Journal of the American Medical Association. Published online 2013. doi:10.1001/jama.2013.280386

72. FDA. FDA Drug Safety Communication: FDA evaluating risk of stroke, heart attack and death with FDA-approved testosterone products. FDA(USA). Published online 2014.

73. Health Canada: Information Update - Possible cardiovascular problems associated with testosterone products. Published 2014. https://healthycanadians.gc.ca/recallalert-rappel-avis/hc-sc/2014/40587a-eng.php

74. Calof OM, Singh AB, Lee ML, et al. Adverse events associated with testosterone replacement in middle-aged and older men: A meta-analysis of randomized, placebocontrolled trials. Journals of Gerontology - Series A Biological Sciences and Medical Sciences. Published online 2005. doi:10.1093/gerona/60.11.1451

75. Rendon RA, Mason RJ, Marzouk K, et al. Canadian urological association recommendations on prostate cancer screening and early diagnosis. Canadian Urological Association Journal. Published online 2017. doi:10.5489/cuaj.4888

76. Marbury T, Hamill E, Bachand R, Sebree T, Smith T. Evaluation of the pharmacokinetic profiles of the new testosterone topical gel formulation, TestinTM, compared to AndroGel ${ }^{\circledR}$. Biopharmaceutics and Drug Disposition. Published online 2003. doi:10.1002/bdd.345

77. Saad F, Aversa A, Isidori AM, Zafalon L, Zitzmann M, Gooren L. Onset of effects of testosterone treatment and time span until maximum effects are achieved. European Journal of Endocrinology. Published online 2011. doi:10.1530/EJE-11-0221 


\section{Figures and Tables}

Fig. 1. Hypothalamic-pituitary-gonadal axis. GnRH: gonadotropin releasing hormone; LH: luteinizing hormone.

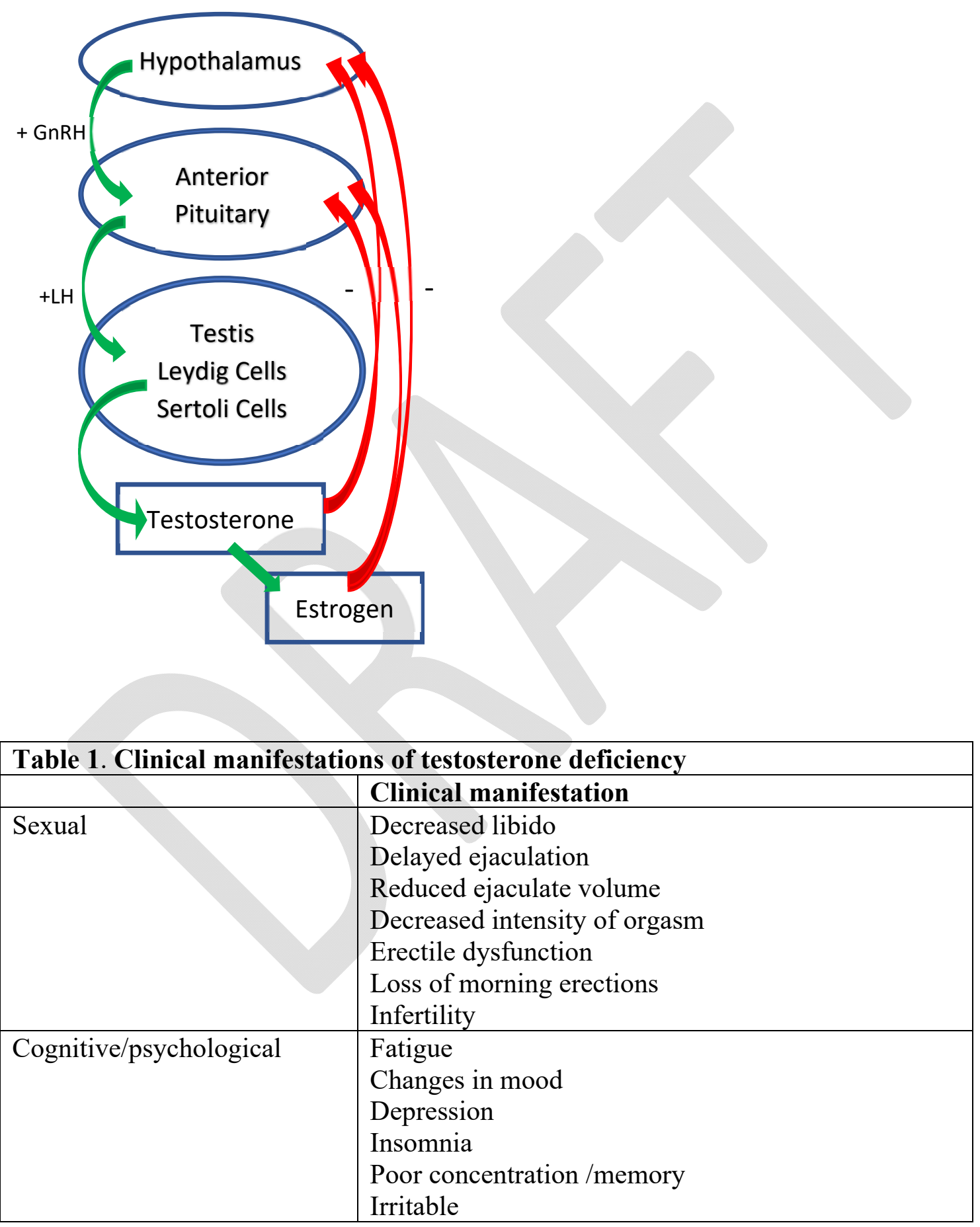




\begin{tabular}{|l|l|}
\hline Physical/structural & Decreased vitality/energy \\
& Anemia \\
& Gynecomastia \\
& Hot flushes \\
& Decrease in muscle mass and strength \\
& Increased visceral body fat \\
& Decreased bone mineral density/osteopenia \\
& Testicular atrophy \\
& Loss of facial, axillary, and pubic hair \\
\hline
\end{tabular}

\begin{tabular}{|l|l|l|}
\hline Table 2. Causes of testosterone deficiency & \\
\hline Medications or substance use & & Opioids \\
& & Glucocorticoids (prednisone) \\
& Chemotherapy \\
& Anticonvulsants \\
& Androgen deprivation therapy \\
& & Exogenous estrogen \\
& & Exogenous testosterone abuse \\
Associated conditions & Marijuana \\
& Acquired & Alcohol abuse \\
& & Diabetes mellitus \\
& Metabolic syndrome \\
& & HIV \\
& & Hyperprolactinemia \\
& & End-stage renal disease \\
& & Liver disease \\
& & Hemochromatosis \\
& & COPD \\
& & Sleep apnea \\
& & Orchitis (mumps) \\
& & Sarcoidosis \\
& & Pituitary tumor/adenoma \\
& & Primary hypothyroidism \\
\hline & Genetic & Kallman's syndrome \\
& & Klinefelter syndrome \\
& & Congenital defect - anorchia, myotonic \\
& dystrophy, cryptorchidism \\
\hline & Radiation \\
& & Trauma \\
& & Nutritional deficiency \\
& Excessive exercise \\
\hline
\end{tabular}




\begin{tabular}{|c|c|c|c|}
\hline Generic name & Trade name & Dosage & Comments \\
\hline $\begin{array}{l}\text { Injectables } \\
\text { Testosterone } \\
\text { Enanthate } \\
\text { Testosterone } \\
\text { Cypionate }\end{array}$ & $\begin{array}{l}\text { Delatestryl } \\
\text { Depo-testosterone }\end{array}$ & $\begin{array}{l}100-200 \mathrm{mg} \text { every } 1-4 \\
\text { weeks } \\
200 \mathrm{mg} \text { every } 2 \text { weeks } \\
\text { or } 100 \mathrm{mg} \text { weekly }\end{array}$ & $\begin{array}{l}\text { Cost-effective } \\
\text { Typically delivered } \\
\text { intramuscularly (IM) into large } \\
\text { muscle including the thigh or } \\
\text { gluteal. May require regular } \\
\text { clinic visits } \\
\text { Wide fluctuations in } \\
\text { testosterone levels requires } \\
\text { mid-cycle testosterone } \\
\text { monitoring } \\
\text { Higher risk for polycythemia }\end{array}$ \\
\hline $\begin{array}{l}\text { Oral medication } \\
\text { Testosterone } \\
\text { Undecanoate }\end{array}$ & Andriol & $\begin{array}{l}40 \text { mg capsules } \\
\text { Initial dose of } 120-160 \\
\text { mg per day in } 2 \text { divided } \\
\text { doses }\end{array}$ & $\begin{array}{l}\text { Absorption } \\
\text { enhanced with fat-rich diet } \\
\text { Short half-life requires multiple } \\
\text { daily dosing } \\
\text { Clinical and biochemical } \\
\text { variability }\end{array}$ \\
\hline $\begin{array}{l}\text { Transdermal } \\
\text { Testosterone patch } \\
\text { Testosterone gels }\end{array}$ & $\begin{array}{l}\text { Androgel } \\
\text { Testim }\end{array}$ & $\begin{array}{l}2.5 \text { or } 5 \mathrm{mg} \text { per day } \\
5-10 \mathrm{~g} \text { per day }\end{array}$ & $\begin{array}{l}\text { Rash/skin irritation common } \\
\text { (patch) } \\
\text { Transfer of medication to } \\
\text { intimate contact (gel) } \\
\text { Variable absorption }\end{array}$ \\
\hline Tans-nasal gel & Natesto $4.5 \%$ & $\begin{array}{l}5.5 \mathrm{mg} \text { ( } 1 \text { pump from } \\
\text { the actuator device) } \\
\text { applied to each nostril } \\
\text { ( } 11 \mathrm{mg} \text { total), two times } \\
\text { daily, at least } 6 \text { hours } \\
\text { apart. Total daily dose } \\
\text { of } 22 \mathrm{mg} \text {. }\end{array}$ & $\begin{array}{l}\text { Approved by Health Canada } \\
\text { but currently unavailable due to } \\
\text { a manufacturing modification } \\
\text { Potentially less suppression of } \\
\text { spermatogenesis }\end{array}$ \\
\hline
\end{tabular}

Adapted from published product monographs for Androgel, Testim, Natesto, Androderm, Andriol, Delatestryl, Depo-testosterone. 


\begin{tabular}{|l|c|c|c|c|}
\hline \multicolumn{6}{|l|}{ Table 4. Testosterone therapy monitoring schedule } \\
\hline & Baseline & 3 months & 6 months & Yearly \\
\hline Symptom evaluation & $\mathrm{X}$ & $\mathrm{X}$ & $\mathrm{X}$ & $\mathrm{X}$ \\
\hline $\begin{array}{l}\text { Adverse event } \\
\text { monitoring }\end{array}$ & & $\mathrm{X}$ & $\mathrm{X}$ & $\mathrm{X}$ \\
\hline Serum testosterone & $\mathrm{X}$ & $\mathrm{X}$ & $\mathrm{X}$ & $\mathrm{X}$ \\
\hline Hematocrit & $\mathrm{X}$ & $\mathrm{X}$ & & $\mathrm{X}$ \\
\hline $\begin{array}{l}\text { Prostate-specific antigen } \\
\text { (PSA) }\end{array}$ & $\mathrm{X}$ & $\mathrm{X}$ & $\mathrm{X}$ & $\mathrm{X}$ \\
\hline $\begin{array}{l}\text { Digital rectal } \\
\text { examination (DRE) }\end{array}$ & $\mathrm{X}$ & & & $\mathrm{X}$ \\
\hline
\end{tabular}

